\title{
The natural history of thin melanoma and the utility of sentinel lymph node biopsy
}

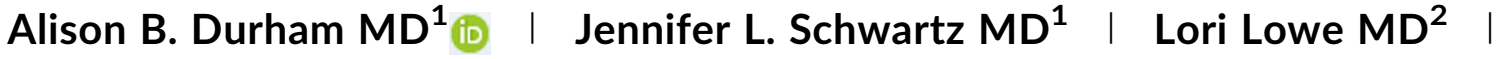 \\ Lili Zhao PhD ${ }^{3}$ | Andrew G. Johnson BA ${ }^{4}$ | Kelly L. Harms MD, PhD ${ }^{1}$ | \\ Christopher K. Bichakjian MD ${ }^{1}$ | Amy P. Orsini MD ${ }^{1}$ | Scott A. McLean MD, PhD \\ Carol R. Bradford MD ${ }^{5}$ | Mark S. Cohen MD ${ }^{6}$ | Timothy M. Johnson MD ${ }^{1,5,6}$ । \\ Michael S. Sabel MD ${ }^{6}$ Sandra L. Wong MD, MS
}

${ }^{1}$ Department of Dermatology, University of Michigan Health System, Ann Arbor, Michigan

${ }^{2}$ Department of Pathology, University of Michigan Health System, Ann Arbor, Michigan

${ }^{3}$ Department of Biostatistics, University of Michigan Health System, Ann Arbor, Michigan

4 University of Michigan Medical School, Ann Arbor, Michigan

5 Department of Otolaryngology - Head and Neck Surgery, University of Michigan Health System, Ann Arbor, Michigan

${ }^{6}$ Department of Surgery, University of Michigan Health System, Ann Arbor, Michigan

7 Department of Surgery, DartmouthHitchcock Medical Center and Geisel School of Medicine at Dartmouth, Lebanon, New Hampshire

\section{Correspondence}

Alison B. Durham MD, University of Michigan Health System, Department of Dermatology, 1500 E. Medical Center Dr., UH South Rm F7672, Ann Arbor, MI 48109-5218.

Email: ambates@med.umich.edu
Background and Objectives: Current literature may overestimate the risk of nodal metastasis from thin melanoma due to reporting of data only from lesions treated with SLNB. Our objective was to define the natural history of thin melanoma, assessing the likelihood of nodal disease, in order to guide selection for SLNB.

Methods: Retrospective review. The primary outcome was the rate of nodal disease. Clinicopathologic factors were evaluated to find associations with nodal disease.

Results: Five hundred and twelve lesions, follow up available for 488 (median: 48 months). Lesions treated with WLE/SLNB compared to WLE alone were more likely to have high-risk features. The rate of nodal disease was higher in the WLE/SLNB group (24 positive SLNB, five false-negative SLNB with nodal recurrence: $10.2 \%$ ) compared to WLE alone (four nodal recurrences: $2.0 \%$ ). Univariate analysis showed age $\leq 45$, Breslow depth $\geq 0.85 \mathrm{~mm}$, mitotic rate $>1 \mathrm{~mm}^{2}$, and ulceration were associated with nodal disease. Multivariate analysis confirmed the association of age $\leq 45$ and ulceration.

Conclusions: SLNB for melanoma 0.75-0.99 mm should be considered in patients age $\leq 45$, Breslow depth $\geq 0.85 \mathrm{~mm}$, mitotic rate $>1 \mathrm{~mm}^{2}$, and/or with ulceration. Thin melanoma $<0.85 \mathrm{~mm}$ without high-risk features may be treated with WLE alone.

\section{KEYWORDS}

melanoma, sentinel lymph node biopsy, thin melanoma

\section{1 | INTRODUCTION}

Thin melanoma is defined as invasive melanoma with Breslow depth $<1.0 \mathrm{~mm}$ and generally has an excellent prognosis. ${ }^{1,2}$ While the risk of nodal and distant metastasis from thin melanoma is low, optimizing treatment is important as an estimated 40000 new cases of thin melanoma are diagnosed annually in the United States alone, representing the majority of new cases. ${ }^{3}$

Robust data support the utility of sentinel lymph node biopsy (SLNB) for melanoma $\geq 1.0 \mathrm{~mm}$ Breslow depth. ${ }^{4-9}$ Data regarding SLNB for thin melanoma $(<1.0 \mathrm{~mm}$ ), in contrast, is limited by retrospective and often conflicting reports of what constitutes high-risk features for nodal 
metastasis and thus selection criteria for performance of SLNB. ${ }^{10,11}$ The 2016 National Comprehensive Cancer Network (NCCN) guidelines recommend "discussion and consideration" of SLNB for melanoma 0.76-1.0 mm, particularly for lesions with high-risk histologic features. ${ }^{8}$ High-risk features can include increasing Breslow depth, ulceration, lymphovascular invasion, and a high mitotic rate. ${ }^{8}$ Other reports have included young age, positive deep margin on biopsy, Clark level IV/V, vertical growth phase, regression, and lack of tumor infiltrating lymphocytes as adverse prognosticators. ${ }^{12-25}$

The purpose of our study was to define the natural history of thin melanoma 0.75-0.99 mm, identify features associated with a higher risk for regional nodal disease, and thus determine patient selection criteria for SLNB in the future. We evaluated a cohort of consecutive patients with melanoma $0.75-0.99 \mathrm{~mm}$ treated at a single institution concordant with NCCN guidelines with either WLE plus SLNB or WLE alone. By analyzing all patients, rather than only patients treated with WLE plus SLNB as in the majority of reports in the literature, we aimed to more completely define the natural history through recurrence outcomes and to identify high-risk features associated with regional nodal disease not simply SLN positivity.

\section{2 | MATERIALS AND METHODS}

Study approval was granted by the University of Michigan Medical School Institutional Review Board for Human Subject Research. Our prospectively collected database was queried for melanoma Breslow depth $0.75-0.99 \mathrm{~mm}$, diagnosed and treated at the University of Michigan between January, 2005 and July, 2015. Cases were excluded if subsequent excisional biopsy or WLE specimen contained melanoma $\geq 1.0 \mathrm{~mm}$ or if the patient had a known second primary Breslow depth $\geq 1.0 \mathrm{~mm}$. Demographic, clinical, and outcome measures were confirmed via the electronic medical record and by phone contact with the patient or referring physician's office. The follow up time for each patient was calculated as the difference between initial biopsy date and date of last contact, with the median follow-up time reported. The follow up period ended April 15, 2016.

All patients were seen in the Multidisciplinary Melanoma Clinic for consultation and discussion regarding melanoma treatment. SLNB was considered for all patients based on Breslow depth 0.75-0.99 mm, concordant with NCCN guidelines. ${ }^{26}$ The presence of additional features considered higher risk for occult regional lymph node metastasis based on current literature included: increasing Breslow depth, ulceration, lymphovascular invasion, high(er) mitotic rate, and young(er) age, regression (defined as partial or complete replacement of melanoma with a variable host response, as previously described), ${ }^{27}$ and positive deep margin on biopsy. Patients were treated with WLE or WLE plus SLNB based on the discussion of potential risks and benefits of the surgery, risk of nodal metastasis, and individual patient preference.

Surgery was performed by 32 different surgeons from the University of Michigan Departments/Divisions of Dermatology, Surgical Oncology, Plastic Surgery, Otolaryngology-Head and Neck
Surgery, Gynecology Oncology (one case), and Pediatric Surgery (one case). Those treated with WLE plus SLNB were treated by one of 14 surgeons who routinely perform SLNB, according to our standard practices. $^{6,8}$ Patients treated with WLE only usually had procedures performed with local anesthesia in a treatment room with a $1 \mathrm{~cm}$ margin. ${ }^{8}$ All WLE specimens were processed using formalin-fixed permanent sections. SLNs were formalin fixed, serially sectioned and evaluated with hematoxylin and eosin, S100, and Melan A immunostains, as previously described. ${ }^{28}$ All specimens were interpreted by dermatopathologists with expertise in melanoma and SLN evaluation.

Patients with a positive $\mathrm{SLN}(\mathrm{s})$ were counseled regarding completion lymph node dissection (CLND) as the standard of care following identification of a positive SLN. Adjuvant therapy was considered following consultation with attending physicians from Medical Oncology with expertise in melanoma. Adjuvant and systemic therapy options did change during the study time frame due to the development of new therapies. ${ }^{29}$

\section{3 | STATISTICAL METHOD}

The outcomes evaluated were: SLNB positivity rate, local recurrence, in-transit recurrence, regional nodal recurrence, distant recurrence, and death from melanoma. Descriptive statistics were calculated for each clinical and pathological variable (frequency/percentage for a categorical variable, mean/standard deviation for a continuous variable). The events of interest were performance of SLNB (yes/no) and presence of nodal disease (defined as either a positive SLNB or nodal recurrence in the follow up period, regardless of SLNB status). To determine an association between any factor and the event of interest, a logistic regression model was used. To appropriately control for potential confounding clinical and pathologic variables when explaining nodal disease, a multivariate logistic regression model was used. All variables were considered in the model, including the two-way interactions (age $\times$ mitotic rate, age $\times$ Breslow depth, and age $\times$ ulceration). A stepwise variable selection procedure was used to select important variables to be included in the final logistic regression model (a significance level of 0.3 was used to allow a variable into the model, and a significance level of 0.35 was used for a variable to stay in the model). The final model included age, Breslow depth, ulceration, and mitotic rate (no interaction was found to be statistically significant). The parameter estimates from the model, the $P$ value from the Wald chi-square test for the significance of the parameter, the odds ratio (OR), and a $95 \%$ Wald-based confidence interval $(95 \% \mathrm{Cl})$ for the OR were reported. Significance was determined if $P<0.05$. For the univariate and multivariate analyses of features associated with the presence of nodal disease, age and Breslow depth were analyzed as categorical variables for consideration as potential patient selection criteria for SLNB in clinical practice guidelines. Consistent with current literature and our practice guidelines, categorical age was defined as $\leq 45$ and $>45$ years. Similarly, and with consideration of the new AJCC definition of $\mathrm{T} 1 \mathrm{a} / \mathrm{b}$ lesions based on a $0.8 \mathrm{~mm}$ cutpoint 
(to define tumor thickness measurements at the "tenth" rather than "hundredth" digit), categorical Breslow depth was defined as $<0.85$ and $\geq 0.85 \mathrm{~mm}$ to allow for classifying as 0.8 or $\geq 0.9 \mathrm{~mm}$, respectively. All analyses were conducted using SAS (version 9.4, SAS Institute, Cary, NC).

\section{4 | RESULTS}

Based on initial biopsy, 552 thin melanomas with Breslow depth $0.75-0.99 \mathrm{~mm}$ were identified. Forty lesions were excluded after subsequent excisional biopsy or WLE demonstrated depth $\geq 1.0 \mathrm{~mm}$. In $24 / 40$ (60\%), residual tumor was noted at the consultation visit and deeper melanoma was suspected. The median final Breslow depth of these 40 lesions was $2.07 \mathrm{~mm}$.

In the study cohort, there were 510 patients with 512 lesions Breslow depth $0.75-0.99 \mathrm{~mm}$. The mean patient age was 56.7 years (range 16-92). Two hundred twenty-six (44.3\%) were women, 284 (55.7\%) were men. The majority of lesions were located on the extremities $(238 / 512,46.5 \%)$ and trunk $(178 / 512,34.8 \%)$ with a smaller number on the head or neck (96/512, 18.8\%). The predominant histologic subtype was superficial spreading (405/512, 79.1\%). The other main histologic subtypes included lentigo maligna melanoma (32/512, 6.3\%), unclassified type (26/512, 5.1\%), nodular (20/512, $3.9 \%)$, nevoid (12/512, 2.3\%), and spitzoid (9/512, 1.8\%).

Two hundred ninety-five (57.6\%) tumors were treated with WLE plus SLNB. The remaining 217 (42.4\%) tumors were treated with WLE alone. Comparison of patient and lesion characteristics for the WLE plus SLNB versus WLE groups showed that younger age (continuous) $(P<0.001)$, gender ( $F$ vs $M)(P<0.001)$, Breslow depth (continuous) $(P<0.0001)$, mitotic rate $\geq 1 \mathrm{~mm}^{2}(P<0.001)$, positive deep margin on biopsy $(P=0.019)$, ulceration $(P=0.007)$, and regression $(P=0.006)$ were associated with performance of SLNB (Table 1).

The SLN identification rate was 98.3\% (290/295). The median number of SLNs removed per patient was 2. Two hundred fifty-four patients (87.6\%) had SLNs removed from only one nodal basin, 34 lesions (11.7\%) mapped to two basins, and two lesions $(0.7 \%)$ mapped to three unique nodal basins. The rate of SLNB positivity was $8.1 \%(24 / 295)$. Twenty-one patients (87.5\%) had one positive SLN, one patient $(4.2 \%)$ had two, and two patients $(8.3 \%)$ had three positive SLNs. No extracapsular extension was identified. Nineteen (79.2\%) of 24 patients with a positive SLNB underwent CLND. Three patients declined and

TABLE 1 Factors associated with performance of SLNB in patients with thin $(0.75-0.99 \mathrm{~mm})$ melanoma

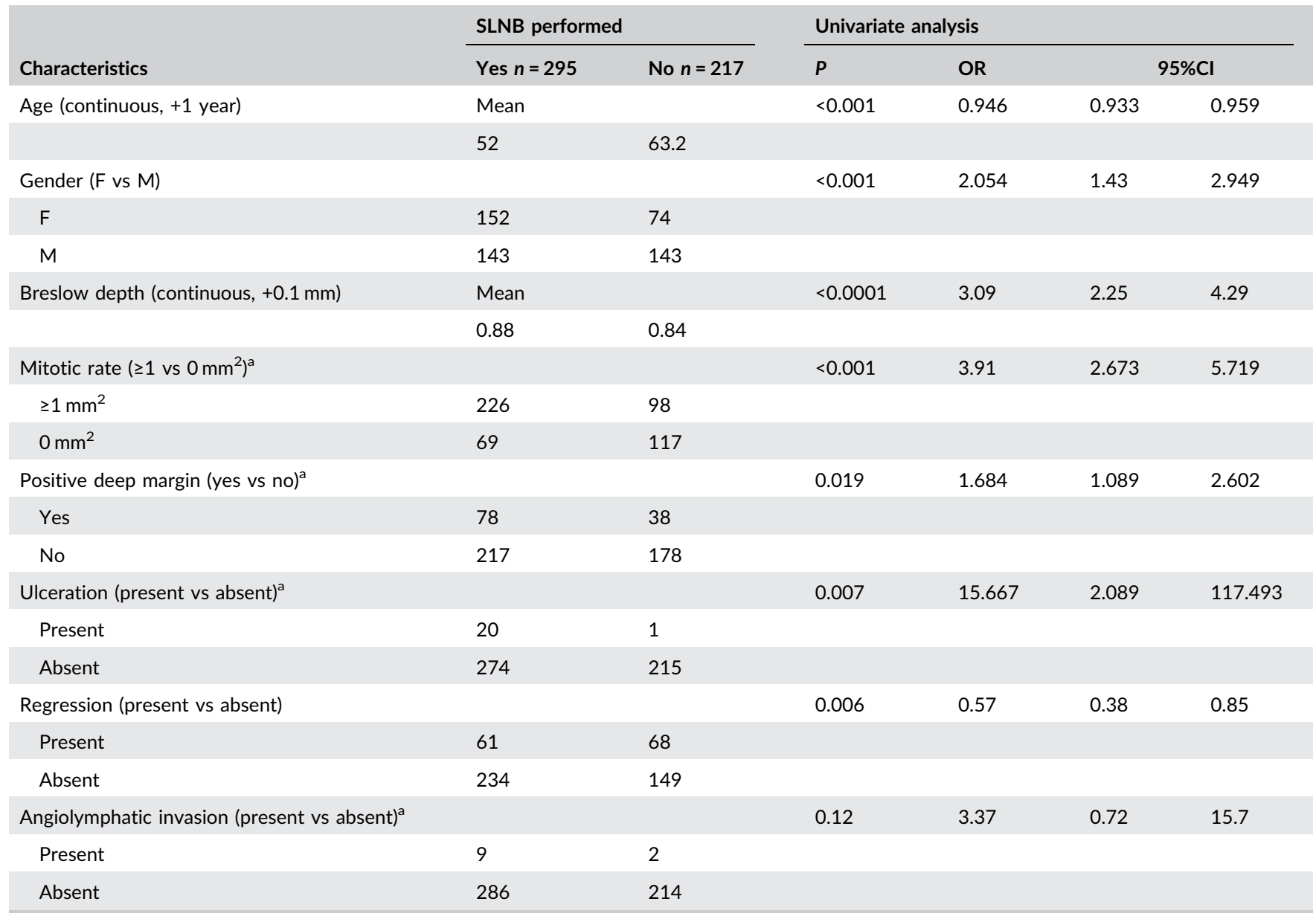

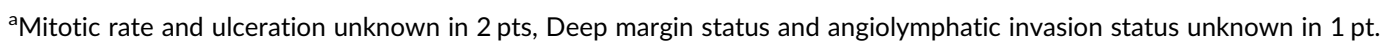


two died prior to CLND from unrelated causes (acute cerebrovascular accident and pyelonephritis). Of the 19 who had CLND, no additional positive nodes were identified.

Following CLND, four patients were treated with adjuvant highdose interferon therapy. Two discontinued treatment due to side effects (after 2 and 3 months, respectively). Another patient entered an adjuvant therapy clinical trial (Dabrafenib $150 \mathrm{mg}$ p.o. twice daily + Trametinib $2 \mathrm{mg}$ p.o. daily vs placebo).

Twenty-four patients (4.7\%) were lost to follow up after the immediate post-operative period (12 WLE, 12 WLE plus SLNB). The median follow-up time for the remaining 486 patients (488 tumors: 205 WLE, 283 WLE plus SLNB) was 48 months. The treatment and outcomes of the cohort are represented in Fig. 1.

Two tumors (0.4\%) located on the head and neck (one lentigo maligna melanoma, one superficial spreading type) locally recurred after 15 and 28 months, respectively, after WLE only. Two patients (0.4\%) developed in-transit recurrence. One patient (treated with WLE alone) was diagnosed concurrently with in-transit and nodal recurrence. The other patient (treated with WLE plus SLNB) developed in-transit recurrence at 52 months and nodal disease at 53 months.

Nine (1.8\%) regional nodal basin recurrences developed; four in patients treated with WLE alone and five in patients treated with WLE plus negative SLNB. Thus, the false negative rate (FNR) was $17.2 \%$ (five false negative SLNB/[five false negative SLNB +24 true positive SLNB]. All five patients with a negative SLNB who developed regional nodal recurrence failed in the same basin as the SLNB. For the two cases of false-negative SLNB on the head and neck, one patient was treated prior to routine use of single-photon emission computed tomography with $\mathrm{CT}$ (SPECT/CT) and, therefore, did not have
SPECT/CT imaging as part of the SLNB procedure. Two patients developed in-transit recurrence in addition to nodal recurrence (one WLE alone [concurrent in-transit and nodal recurrence]), one WLE plus SLNB [in-transit recurrence one month before nodal recurrence]). The median time to nodal recurrence was 16 months (range: 6-53). No nodal recurrences occurred in patients who had a positive SLNB (median follow up time for this subset of patients was 32.5 months), including those who did not have CLND. The patient and lesion characteristics for cases of nodal recurrence are provided in Table 2.

In total, 33 (6.8\%) patients ultimately developed nodal metastases from thin melanoma (24 found with positive SLNB and nine nodal recurrences). Regional nodal disease was the most common first site of disease identified beyond the primary site (32 [one with concurrent in-transit disease and one with concurrent distant disease] of 35 patients with stage III/IV disease). Univariate analysis showed that age $\leq 45(P=0.027)$, Breslow depth $\geq 0.85 \mathrm{~mm}(P=0.04)$, mitotic rate $>1 \mathrm{~mm}^{2} \quad(P=0.031)$, and ulceration $(P=0.001)$ were significantly associated with nodal disease. Microsatellitosis was not present in any tumor. Multivariate analysis was performed as previously described. The final model considered age (>45 vs $\leq 45$ ), Breslow depth ( $\geq 0.85 \mathrm{vs}<0.85 \mathrm{~mm}$ ), mitotic rate ( $>1 \mathrm{vs} \leq 1 \mathrm{~mm}^{2}$ ), and ulceration (present vs absent); only age $\leq 45$ (>45 vs $\leq 45$ : $P=0.007$, OR 0.336 , $95 \% \mathrm{Cl} 0.152-0.74$ ) and ulceration (present vs absent: $P=0.003$, OR $5.932,95 \% \mathrm{Cl} 1.805-19.496)$ were statistically significant independent factors associated with nodal disease (Table 3 ).

Eight (1.6\%) distant recurrences developed (the median time to distant recurrence was 28.5 months, range 10-74), resulting in seven deaths. Four lesions were treated with WLE and four were treated with WLE plus negative SLNB. Nodal recurrence preceded distant

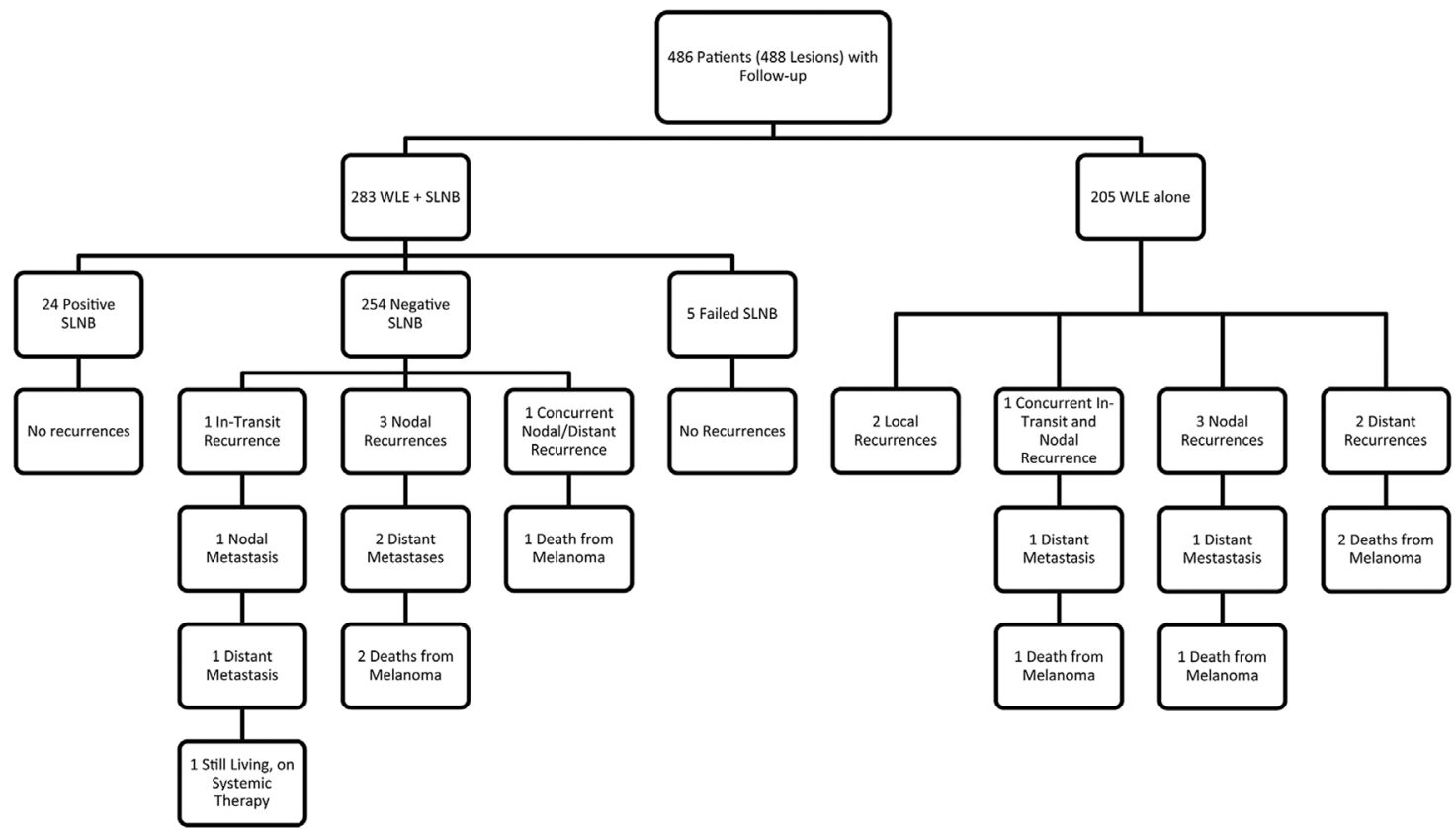

FIGURE 1 Outcome measures. This figure shows outcome measures including: local recurrence, in-transit recurrence, nodal metastasis, distant metastasis, and death from melanoma for 486 patients with 488 primary lesions 0.75-0.99 mm Breslow depth after a median follow up time of 48 months. 
TABLE 2 Patient and lesion characteristics in cases of nodal recurrence

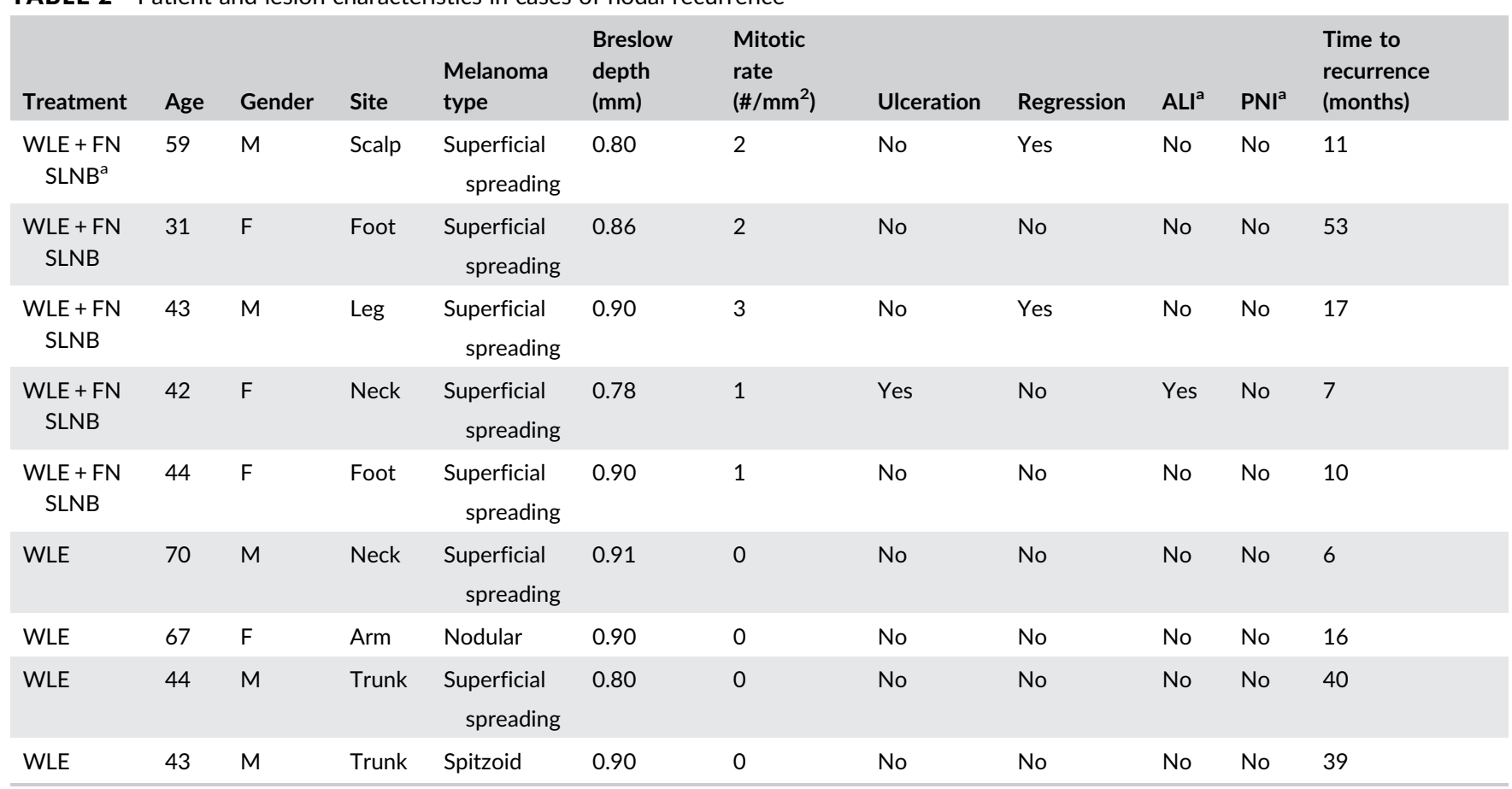

${ }^{a}$ WLE, wide local excision; FN SLNB, false-negative sentinel lymph node biopsy; ALI, angiolymphatic invasion; PNI, perineural invasion.

metastasis in five cases (62.5\%), by a median time of 12 months (range: 4-21). One patient developed nodal recurrence and distant metastasis concurrently after 10 months (WLE and false-negative SLNB). Two patients developed distant metastatic disease without nodal recurrence (both were treated with WLE alone). One patient with distant metastasis remains alive (78 months from primary diagnosis, 23 months from initiation of systemic therapy). No distant recurrences developed in patients with a positive SLNB (median follow up time for this subset of patients was 32.5 months).

\section{5 | DISCUSSION}

The intent of this study was to use SLNB data and recurrence outcomes to define the natural history of thin melanoma $(0.75-0.99 \mathrm{~mm})$ in terms of disease recurrence and risk of nodal metastases with and without SLNB, and to identify factors associated with nodal disease that could be useful as patient selection criteria for SLNB. This study was intentionally designed to include patients treated with WLE plus SLNB and patients treated with WLE alone and differs from the majority of outcome studies of thin melanoma that only evaluate patients undergoing WLE plus SLNB. As patients are frequently selected for SLNB because of higher risk features for nodal metastases, these prior studies are inherently biased and may overestimate the likelihood of nodal metastases from thin melanoma.

Outcomes from this study showed that regional nodal disease was the most common first site of disease identified beyond the primary site (24 patients with positive SLNB, eight patients with delayed nodal recurrence [one with concurrent in-transit recurrence, one with concurrent distant recurrence]). Five of the eight patients with nodal recurrence subsequently died of distant disease. Only two patients developed distant disease as the first site of disease beyond the primary site and both patients died from melanoma.

In our cohort of all patients with thin melanoma, Breslow depth $0.75-0.99 \mathrm{~mm}$, the overall nodal metastatic rate was $6.8 \%$ (33 nodal metastases/488 tumors). The nodal metastatic rate observed in patients treated with WLE plus SLNB (24 positive SLNB, five falsenegative SLNB with nodal recurrence) was $10.2 \%$. In patients treated with WLE alone, the rate of nodal disease was $2.0 \%$ (four nodal recurrences). Compared to patients treated with WLE only, patients who underwent SLNB were more likely to be younger, female, have a deeper Breslow depth, mitotic rate $\geq 1 \mathrm{~mm}^{2}$, positive deep margin, ulceration, and/or regression. Notably, all four of the patients treated with WLE alone that had nodal recurrence had primary lesions with Breslow depth $\geq 0.8 \mathrm{~mm}(0.91,0.9,0.9$, and $0.8 \mathrm{~mm})$ and two patients were $<45$ years old ( 43 and 44 ) (Table 2 ).

The higher rate of nodal disease in the SLNB group may be, at least partly, attributable to lead-time bias and a relatively limited follow up time. Thin melanoma has been reported to recur long after initial treatment, in some cases $>10$ years after diagnosis. ${ }^{15}$ It is likely that additional recurrences will develop in all groups, including those patients with a negative SLNB and those treated with WLE alone. Continued follow-up of our cohort beyond the reported median 48 months will provide additional valuable information.

Interestingly, the largest meta-analysis (60 studies, 10928 patients) to evaluate SLNB for thin melanoma was published in 2016 and included only those who had SLNB. Breslow depth 
TABLE 3 Factors associated with nodal disease in patients with thin $(0.75-0.99 \mathrm{~mm})$ melanoma

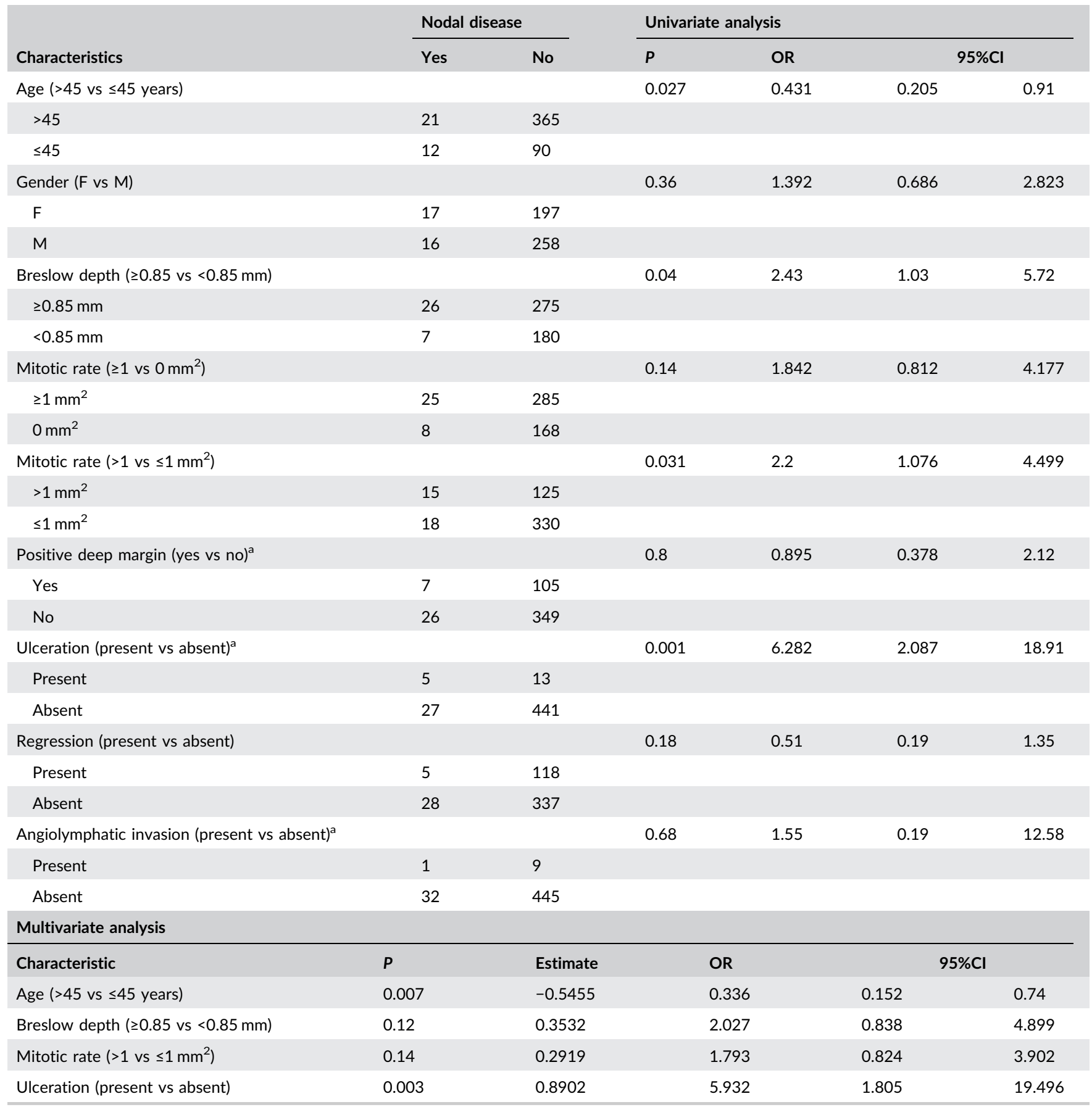

aUlceration status unknown in 2 pts, Deep margin status and angiolymphatic invasion unknown in 1 pt.

$\geq 0.75 \mathrm{~mm}$ (adjusted odds ratio (AOR) 1.9; $95 \% \mathrm{Cl} 1.08-3.340$ ), Clark level IV/V (AOR 2.24; 95\%Cl 1.23-4.08), mitotic rate $\geq 1 \mathrm{~mm}^{2}$ (AOR $6.64 ; 95 \% \mathrm{Cl}$ 2.77-15.88), and presence of microsatellites (unadjusted OR $6.94 ; 95 \% \mathrm{Cl} 2.13-22.60$ ) were associated with a positive SLNB. The authors concluded that patients with melanoma $\geq 0.75 \mathrm{~mm}$ should be offered SLNB, based on a SLNB positivity likelihood of $8.8 \%$. If other high-risk features are present, the rate of SLNB positivity may be even higher. $^{11}$

Univariate analysis of our entire cohort, including patients treated with WLE alone, showed age $\leq 45$, Breslow depth $\geq 0.85 \mathrm{~mm}$, mitotic rate
$>1 \mathrm{~mm}^{2}$, and ulceration to be significantly associated with nodal disease (positive SLNB or nodal recurrence in the follow up period) supporting the use of these risk features as selection criteria for SLNB in patients with thin melanoma $0.75-0.99 \mathrm{~mm}$. This analysis is more clinically relevant than prior reports because it uses the entire population of patients with thin melanoma rather than just the subset of those selected for SLNB. Our data would suggest that SLNB be considered for AJCC 8th Edition ${ }^{30}$ T1b lesions 0.8-1.0 $\mathrm{mm}$ with any of the following high-risk features: age $\leq 45$, Breslow depth $\geq 0.85 \mathrm{~mm}$ (rounded to $0.9 \mathrm{~mm}$ for the AJCC 8th Edition), mitotic rate $>1 \mathrm{~mm}^{2}$, and/or ulceration. 
In thin melanoma there is conflicting evidence regarding the prognostic significance of a positive SLN. ${ }^{2,15,23,31}$ In our patients with a positive SLNB, no recurrences were noted in the follow up period. It is possible that the follow up time is insufficient to capture long-term events. However, this observation also raises the possibility that SLNB-directed early intervention may provide a therapeutic benefit with improved outcomes in thin melanoma similar to that demonstrated for intermediate depth melanoma in the Multicenter Selective Lymphadenectomy-I (MSLT-I) trial. ${ }^{9}$ In our patients with a positive SLNB who underwent CLND (19/24 patients), no additional positive nodes were identified. Results from the Multicenter Selective Lymphadenectomy Trial-II and adjuvant therapy trials may lead to changes in the management of SLNB positive patients in the future. ${ }^{32}$

Our study population may be subject to potential selection bias based on patient referral to a tertiary cancer center. However, our Multidisciplinary Melanoma program evaluates and treats nearly $80 \%$ of all melanoma cases in our state. Additional limitations include the retrospective design and relatively limited follow up time, though a median follow up of $\mathbf{4 8}$ months is equivalent to or longer than many comparable studies reported in contemporary literature.

\section{6 | CONCLUSIONS}

Patients with thin melanoma can and do develop regional lymph node and distant disease and may die from melanoma. The eight distant recurrences and seven deaths serve as a reminder that although thin melanoma has an excellent prognosis, some patients will have an adverse outcome. This study supports that regional nodal disease is the most common first site of spread detected beyond the primary site in the natural history of thin melanoma $0.75-0.99 \mathrm{~mm}$. Furthermore, this study supports that a subset of these thin melanomas have a sufficient risk to consider nodal staging with SLNB. Specifically, SLNB should be strongly considered for thin melanoma $0.75-0.99 \mathrm{~mm}$ in the setting of patient age $\leq 45$ years, Breslow depth $\geq 0.85 \mathrm{~mm}$, mitotic rate $>1 \mathrm{~mm}^{2}$, and/or ulceration of the primary lesion. Thin melanoma $<0.85 \mathrm{~mm}$ (to be defined as $\leq 0.8 \mathrm{~mm}$ in the 8th edition of the AJCC) without additional high-risk features likely has a low rate of nodal metastases and therefore may be treated with WLE alone.

\section{ACKNOWLEDGMENT}

We are indebted to Nisha Meireles, Clinical Research Specialist, Multidisciplinary Cutaneous Oncology Program, for database management.

\section{REFERENCES}

1. Balch CM, Gershenwald JE, Soong SJ, et al. Final version of 2009 AJCC melanoma staging and classification. J Clin Oncol. 2009;27: 6199-6206.

2. Cooper C, Wayne JD, Damstetter EM, et al. A 10-year, singleinstitution analysis of clinicopathologic features and sentinel lymph node biopsy in thin melanomas. J Am Acad Dermatol. 2013;69: 693-699.

3. Andtbacka RH, Gershenwald JE. Role of sentinel lymph node biopsy in patients with thin melanoma. J Natl Compr Canc Netw. 2009;7: 308-317.

4. Morton DL, Wen DR, Wong JH, et al. Technical details of intraoperative lymphatic mapping for early stage melanoma. Arch Surg. 1992;127:392-399.

5. Garbe C, Hauschild A, Volkenandt M, et al. Evidence and interdisciplinary consensus-based German guidelines: surgical treatment and radiotherapy of melanoma. Melanoma Res. 2008;18:61-67.

6. Bichakjian CK, Halpern AC, Johnson TM, et al. Guidelines of care for the management of primary cutaneous melanoma. American Academy of Dermatology. J Am Acad Dermatol. 2011;65:1032-1047.

7. Wong SL, Balch CM, Hurley P, et al. Sentinel lymph node biopsy for melanoma: American Society of Clinical Oncology and Society of Surgical Oncology joint clinical practice guideline. Ann Surg Oncol. 2012;19:3313-3324.

8. Coit DG. NCCN Clinical Practice Guidelines in Oncology (NCCN Guidelines). 2016; Melanoma. Version I. 2016.

9. Morton DL, Thompson JF, Cochran AJ, et al. Final trial report of sentinel-node biopsy versus nodal observation in melanoma. $\mathrm{N} \mathrm{Engl} \mathrm{J}$ Med. 2014;370:599-609.

10. Durham AB, Wong SL. Sentinel lymph node biopsy in melanoma: controversies and current guidelines. Future Oncol. 2014;10:429-442.

11. Cordeiro E, Gervais MK, Shah PS, Look Hong NJ, Wright FC. Sentinel lymph node biopsy in thin cutaneous melanoma: a systematic review and meta-analysis. Ann Surg Oncol. 2016;23:4178-4188.

12. Vermeeren L, Van der Ent F, Sastrowijoto P, Hulsewe K. Sentinel lymph node biopsy in patients with thin melanoma: occurrence of nodal metastases and its prognostic value. Eur J Dermatol. 2010;20:30-34.

13. Venna SS, Thummala S, Nosrati M, et al. Analysis of sentinel lymph node positivity in patients with thin primary melanoma. J Am Acad Dermatol. 2013;68:560-567.

14. Mozzillo N, Pennacchioli E, Gandini S, et al. Sentinel node biopsy in thin and thick melanoma. Ann Surg Oncol. 2013;20:2780-2786.

15. Wong SL, Brady MS, Busam KJ, Coit DG. Results of sentinel lymph node biopsy in patients with thin melanoma. Ann Surg Oncol. 2006;13:302-309.

16. Ranieri JM, Wagner JD, Wenck S, Johnson CS, Coleman JJ, 3rd. The prognostic importance of sentinel lymph node biopsy in thin melanoma. Ann Surg Oncol. 2006;13:927-932.

17. Oliveira Filho RS, Ferreira LM, Biasi L, Enokihara MM, Paiva GR, Wagner J. Vertical growth phase and positive sentinel node in thin melanoma. Braz J Med Biol Res. 2003;36:347-350.

18. Olah J, Gyulai R, Korom I, Varga E, Dobozy A. Tumour regression predicts higher risk of sentinel node involvement in thin cutaneous melanomas. Br J Dermatol. 2003;149:662-663.

19. Statius Muller MG, van Leeuwen PA, van Diest PJ, Vuylsteke RJ, Pijpers R, Meijer S. No indication for performing sentinel node biopsy in melanoma patients with a Breslow thickness of less than $0.9 \mathrm{~mm}$. Melanoma Res. 2001;11:303-307.

20. Kesmodel SB, Karakousis GC, Botbyl JD, et al. Mitotic rate as a predictor of sentinel lymph node positivity in patients with thin melanomas. Ann Surg Oncol. 2005;12:449-458.

21. Murali R, Haydu LE, Quinn MJ, et al. Sentinel lymph node biopsy in patients with thin primary cutaneous melanoma. Ann Surg. 2012;255:128-133.

22. Bleicher RJ, Essner R, Foshag LJ, Wanek LA, Morton DL. Role of sentinel lymphadenectomy in thin invasive cutaneous melanomas. J Clin Oncol. 2003;21:1326-1331.

23. Wright $B E$, Scheri RP, Ye X, et al. Importance of sentinel lymph node biopsy in patients with thin melanoma. Arch Surg. 2008;143:892-899, discussion 9-900. 
24. Koshenkov VP, Shulkin D, Bustami R, Chevinsky AH, Whitman ED. Role of sentinel lymphadenectomy in thin cutaneous melanomas with positive deep margins on initial biopsy. J Surg Oncol. 2012;106: 363-368.

25. Sondak VK, Taylor JM, Sabel MS, et al. Mitotic rate and younger age are predictors of sentinel lymph node positivity: lessons learned from the generation of a probabilistic model. Ann Surg Oncol. 2004;11: 247-258.

26. Coit D. NCCN Clinical Practice Guidelines in Oncology (NCCN Guidelines). Version I. 2017.

27. Smoller BR. Histologic criteria for diagnosing primary cutaneous malignant melanoma. Mod Pathol. 2006;19:S34-S40.

28. Ghaferi AA, Wong SL, Johnson TM, et al. Prognostic significance of a positive nonsentinel lymph node in cutaneous melanoma. Ann Surg Oncol. 2009;16:2978-2984.

29. Achkar T, Tarhini AA. The use of immunotherapy in the treatment of melanoma. J Hematol Oncol. 2017;10:88.

30. Gershenwald JE, Scolyer RA, Hess KR, et al. AJCC cancer staging manual. New York, NY: Springer Science + Business Media; 2016.
31. Karakousis G, Gimotty PA, Bartlett EK, et al. Thin melanoma with nodal involvement: analysis of demographic, pathologic, and treatment factors with regard to prognosis. Ann Surg Oncol. 2017; 24:952-959.

32. Morton DL. Overview and update of the phase III multicenter selective Iymphadenectomy trials (MSLT-I and MSLT-II) in melanoma. Clin Exp Metastasis. 2012;29:699-706.

How to cite this article: Durham AB, Schwartz JL, Lowe L, et al. The natural history of thin melanoma and the utility of sentinel lymph node biopsy. J Surg Oncol.

2017;116:1185-1192.

https://doi.org/10.1002/jso.24765 